\title{
Spin transport in intermediate-energy heavy-ion collisions as a probe of in-medium spin-orbit interactions
}

\author{
Yin Xia, ${ }^{1,2}$ Jun $\mathrm{Xu}^{*},{ }^{1}$ Bao-An $\mathrm{Li}^{3,},{ }^{3}$ and Wen-Qing Shen ${ }^{1}$ \\ ${ }^{1}$ Shanghai Institute of Applied Physics, \\ Chinese Academy of Sciences, Shanghai 201800, China \\ ${ }^{2}$ University of Chinese Academy of Sciences, Beijing 100049, China \\ ${ }^{3}$ Department of Physics and Astronomy, \\ Texas A\&M University-Commerce, Commerce, TX 75429-3011, USA \\ ${ }^{4}$ Department of Applied Physics, Xi'an Jiao Tong University, Xi'an 710049, China
}

\begin{abstract}
The spin up-down splitting of collective flows in intermediate-energy heavy-ion collisions as a result of the nuclear spin-orbit interaction is investigated within a spin- and isospin-dependent Boltzmann-Uehing-Uhlenbeck transport model SIBUU12. Using a Skyrme-type spin-orbit coupling quadratic in momentum, we found that the spin splittings of the directed flow and elliptic flow are largest in peripheral $\mathrm{Au}+\mathrm{Au}$ collisions at beam energies of about $100-200 \mathrm{MeV} / \mathrm{nucleon}$, and the effect is considerable even in smaller systems especially for nucleons with high transverse momenta. The collective flows of light clusters of different spin states are also investigated using an improved dynamical coalescence model with spin. Our study can be important in understanding the properties of in-medium nuclear spin-orbit interactions once the spin-dependent observables proposed in this work can be measured.
\end{abstract}

PACS numbers: 25.70.-z, 24.10.Lx, 25.75.Ld, 21.30.Fe, 21.10.Hw

* corresponding author: xujun@sinap.ac.cn 


\section{INTRODUCTION}

Understanding properties of nucleon-nucleon interactions under extreme conditions of spin, isospin, and density is one of the forefronts of both nuclear physics and astrophysics. It is well known that bare nucleon-nucleon interactions are spin-isospin dependent. As a result, nucleon mean-field potentials in nuclear medium are also spin-isospin dependent as shown by various many-body approaches in the literature. On one hand, much progress has been made in recent years in studying effects of the isospin dependence of nuclear interactions on properties of nuclei, nuclear reactions, neutron stars and gravitational waves [1-6], and many interesting questions remain to be addressed [7]. On the other hand, there are also many interesting questions regarding the spin dependence of nuclear interactions to be answered. It is well known that the spin-orbit coupling is one of the most important spin-dependent nuclear interactions and it has many significant effects on the structure of nuclei $[8,9]$. However, detailed properties of the spin-orbit potential, such as its strength, isospin dependence [10], and density dependence [11], are still poorly known and are current topics of intensive investigations. These studies are relevant for our understanding of the structures of rare isotopes and their impacts on astrophysics [12-16]. Moreover, the spin and isospin dependence of nuclear interactions are often intertwined. Thus, the study of spin-isospin correlation is an interesting topic in its own right.

In nuclear reactions, while the spin-dependent interaction has been shown to be critical in understanding some features of few-body reactions within various models, its effects in heavy-ion collisions are less known. Usually only spin-averaged nucleon interactions are used in transport model studies of heavy-ion collisions mostly due to the difficulties in measuring spin-dependent observables. Nevertheless, encouraged by the recent progress in developing spin polarized beams and measuring the analyzing power used to quantify the spin-asymmetry in nuclear reactions, we are optimistic that experimental studies of spin physics with heavy ions will be possible in the near future. For example, spin polarized beams can now be produced with nucleon removal or pickup reactions [17]. In addition, an analyzing power as large as $100 \%$ can be achieved in pp or pA scattering at certain energies and scattering angles $[18,19]$, providing a possible way to disentangle nucleons with different spins in the final state of the reaction. Heavy-ion collisions create nuclear medium of varying density and isospin asymmetry. They may thus be used as a testing ground of in-medium 
properties of nuclear spin-orbit interactions.

To provide a theoretical tool for studying spin physics with heavy-ion reactions, by incorporating the nuclear spin-orbit interaction and the nucleon spin degree of freedom explicitly into an earlier version of nuclear transport model, we have developed the spin- and isospindependent Boltzmann-Uehing-Uhlenbeck transport model (SIBUU12), and studied the spin splitting of transverse flows in intermediate-energy heavy-ion collisions [20-22]. It is worth noting that a similar approach based on the quantum molecular dynamics model was developed more recently in Ref. [23]. In the present work, by incorporating the quantum description of spin dynamics derived in Ref. [24] into the SIBUU12 transport model, we investigate in detail the spin-dependent collective flows of both single nucleons and light clusters in heavy-ion collisions at intermediate energies. We found that the spin up-down splittings of the directed flow and elliptic flow are largest in peripheral $\mathrm{Au}+\mathrm{Au}$ collisions at beam energies of about 100-200 MeV/nucleon, and a larger spin splitting effect is observed in the present work with a quantum description of spin dynamics compared to earlier studies $[20-22]$.

\section{SIBUU12 TRANSPORT MODEL WITH QUANTUM DESCRIPTION OF SPIN DYNAMICS}

In this section, we outline the framework and give the major ingredients of the SIBUU12 transport model using a quantum description of spin dynamics [24], where the nuclear spinorbit coupling and the explicit spin states of nucleons have been incorporated. We begin with the spin-dependent Boltzmann-Vlasov (BV) equation obtained by a Wigner transformation of the Liouville equation for the density matrix [25-27]

$$
\frac{\partial \hat{f}}{\partial t}+\frac{i}{\hbar}[\hat{\varepsilon}, \hat{f}]+\frac{1}{2}\left(\frac{\partial \hat{\varepsilon}}{\partial \vec{p}} \cdot \frac{\partial \hat{f}}{\partial \vec{r}}+\frac{\partial \hat{f}}{\partial \vec{r}} \cdot \frac{\partial \hat{\varepsilon}}{\partial \vec{p}}\right)-\frac{1}{2}\left(\frac{\partial \hat{\varepsilon}}{\partial \vec{r}} \cdot \frac{\partial \hat{f}}{\partial \vec{p}}+\frac{\partial \hat{f}}{\partial \vec{p}} \cdot \frac{\partial \hat{\varepsilon}}{\partial \vec{r}}\right)=0
$$

where the $\hat{\varepsilon}$ and $\hat{f}$ are from the Wigner transformation of the energy and phase-space density matrix, respectively. The $\hat{\varepsilon}$ and $\hat{f}$ can be decomposed into their scalar and vector parts, i.e.,

$$
\begin{aligned}
& \hat{\varepsilon}(\vec{r}, \vec{p})=\varepsilon(\vec{r}, \vec{p}) \hat{I}+\vec{h}(\vec{r}, \vec{p}) \cdot \vec{\sigma}, \\
& \hat{f}(\vec{r}, \vec{p})=f_{0}(\vec{r}, \vec{p}) \hat{I}+\vec{g}(\vec{r}, \vec{p}) \cdot \vec{\sigma},
\end{aligned}
$$


where $\vec{\sigma}=\left(\sigma_{x}, \sigma_{y}, \sigma_{z}\right)$ and $\hat{I}$ are respectively the Pauli matrices and the $2 \times 2$ unit matrix, $\varepsilon$ and $f_{0}$ are the scalar parts of the effective single-particle energy $\hat{\varepsilon}$ and density $\hat{f}$, respectively, and $\vec{h}$ and $\vec{g}$ are the corresponding vector distributions.

Adopting the Skyrme-type effective two-body nuclear spin-orbit interaction [28]

$$
V_{s o}=i W_{0}\left(\vec{\sigma}_{1}+\vec{\sigma}_{2}\right) \cdot \vec{k} \times \delta\left(\vec{r}_{1}-\vec{r}_{2}\right) \vec{k}^{\prime}
$$

with $W_{0}$ being the strength of the spin-orbit coupling, which is set to be $W_{0}=150 \mathrm{MeVfm}^{5}$ in the calculations unless stated otherwise, $\vec{\sigma}_{1(2)}$ being the Pauli matrix, $\vec{k}=\left(\vec{p}_{1}-\vec{p}_{2}\right) / 2$ being the relative momentum operator acting on the right with $\vec{p}=-i \nabla$, and $\vec{k}^{\prime}$ being the complex conjugate of $\vec{k}$, the explicit expressions of the scalar and vector part in Eq. (2) can be calculated from the Skyrme-Hartree-Fock (SHF) method as

$$
\begin{aligned}
& \varepsilon_{q}^{s o}(\vec{r}, \vec{p})=h_{1}+h_{4}, \\
& \vec{h}_{q}^{s o}(\vec{r}, \vec{p})=\vec{h}_{2}+\vec{h}_{3},
\end{aligned}
$$

with

$$
\begin{aligned}
& h_{1}=-\frac{W_{0}}{2} \nabla_{\vec{r}} \cdot\left[\vec{J}(\vec{r})+\vec{J}_{q}(\vec{r})\right], \\
& \vec{h}_{2}=-\frac{W_{0}}{2} \nabla_{\vec{r}} \times\left[\vec{j}(\vec{r})+\vec{j}_{q}(\vec{r})\right], \\
& \vec{h}_{3}=\frac{W_{0}}{2} \nabla_{\vec{r}}\left[\rho(\vec{r})+\rho_{q}(\vec{r})\right] \times \vec{p}, \\
& h_{4}=-\frac{W_{0}}{2} \nabla_{\vec{r}} \times\left[\vec{s}(\vec{r})+\vec{s}_{q}(\vec{r})\right] \cdot \vec{p},
\end{aligned}
$$

and $q=n$ or $p$ being the isospin index. The $\rho, \vec{s}, \vec{j}$, and $\vec{J}$ are respectively the number, spin, momentum, and spin-current densities, and their definitions in terms of the nucleon wave function can be found in Ref. [29]. They can be expressed in terms of the scalar Wigner function $f_{0}$ and the vector Wigner function $\vec{g}$ as

$$
\begin{aligned}
& \rho(\vec{r})=2 \int d^{3} p f_{0}(\vec{r}, \vec{p}), \\
& \vec{s}(\vec{r})=2 \int d^{3} p \vec{g}(\vec{r}, \vec{p}), \\
& \vec{j}(\vec{r})=2 \int d^{3} p \frac{\vec{p}}{\hbar} f_{0}(\vec{r}, \vec{p}), \\
& \vec{J}(\vec{r})=2 \int d^{3} p \frac{\vec{p}}{\hbar} \times \vec{g}(\vec{r}, \vec{p}) .
\end{aligned}
$$


The $\rho$ and $\vec{J}(\vec{s}$ and $\vec{j}$ ) are the time-even (time-odd) densities, and their corresponding contributions are the time-even (time-odd) potentials. Although the time-odd potential is negligible in studying spherical nuclei, it is important in dealing with deformed nuclei. In heavy-ion collisions with all kinds of collision geometries, both the time-even and time-odd potentials should be included. It is also worth noting that the spin-dependent potential from the SHF calculation is quadratic in momentum $\vec{p}$, so is that from the lowest-order nonrelativistic expansion of the Dirac equation based on the relativistic mean-field approach [30]. It can be of great interest to study effects of the momentum dependence of the spin-orbit coupling on the spin dynamics.

In the quantum description of spin dynamics, since the total angular momentum in noncentral heavy-ion collisions is in the $y$ direction perpendicular to the reaction plane, we define the nucleons with spin in $+y(-y)$ direction as the spin-up (spin-down) nucleons. By generalizing the test-particle method [31] for both spin-up and spin-down particles, the spindependent BV equation can be solved numerically, and the equations of motion (EOMs) for the test particles can be written as [24]

$$
\begin{aligned}
& \frac{\partial \vec{r}_{i}}{\partial t}=\frac{\vec{p}_{i}}{m}+\nabla_{\vec{p}_{i}}\left(h_{1}+h_{4}\right)+\nabla_{\vec{p}_{i}}\left(\vec{h}_{2} \cdot \vec{n}_{i}+\vec{h}_{3} \cdot \vec{n}_{i}\right), \\
& \frac{\partial \vec{p}_{i}}{\partial t}=-\nabla_{\vec{r}_{i}} U_{q}-\nabla_{\vec{r}_{i}}\left(h_{1}+h_{4}\right)-\nabla_{\vec{r}_{i}}\left(\vec{h}_{2} \cdot \vec{n}_{i}+\vec{h}_{3} \cdot \vec{n}_{i}\right),
\end{aligned}
$$

with $\vec{n}_{i}=+\hat{y}$ for spin-up nucleons and $-\hat{y}$ for spin-down ones. We note here that an additional kinetic energy term $p^{2} / 2 m$ and a spin-independent potential $U_{q}$ are added to Eq. (5) in deriving the above EOMs, where $U_{q}$ is assumed to be momentum independent and reproduces the empirical saturation properties of nuclear matter. Correspondingly, the number, spin, momentum, and spin-current densities can be calculated from the test-particle method via

$$
\begin{aligned}
& \rho(\vec{r})=\frac{1}{N_{T P}} \sum_{\mathrm{i}} \delta\left(\vec{r}-\vec{r}_{i}\right), \\
& \vec{s}(\vec{r})=\frac{1}{N_{T P}} \sum_{\mathrm{i}} \vec{n}_{i} \delta\left(\vec{r}-\vec{r}_{i}\right), \\
& \vec{j}(\vec{r})=\frac{1}{N_{T P}} \sum_{\mathrm{i}} \frac{\vec{p}_{i}}{\hbar} \delta\left(\vec{r}-\vec{r}_{i}\right), \\
& \vec{J}(\vec{r})=\frac{1}{N_{T P}} \sum_{\mathrm{i}}\left(\frac{\vec{p}_{i}}{\hbar} \times \vec{n}_{i}\right) \delta\left(\vec{r}-\vec{r}_{i}\right),
\end{aligned}
$$

with $N_{T P}$ being the number of test particles per nucleon. 
The initial density distributions of the projectile and target nuclei are obtained from Hartree-Fock calculations using a modified Skyrme interaction [32]. The spins of initial nucleons are assumed to be randomly distributed, i.e., half spin-up and half spin-down, by neglecting the shell effect and the spin of a nucleus. Since the spin of a nucleon may flip after nucleon-nucleon scatterings but its dependence on the energy and isospins of the colliding nucleons is poorly known, to focus on effects from the spin-dependent nuclear mean-field potential we randomize the spin state of nucleons after their successful scatterings. We notice that the spin- and isospin-dependent Pauli blocking is also consistently implemented. Similar to the Stern-Gerlach experiment, nucleons with different spins are expected to have different dynamics according to the spin-dependent EOMs, and this will lead to the spin splitting of the final observables to be discussed in the following.

\section{RESULTS AND DISCUSSIONS}

In this section, we examine effects of the spin-orbit interaction on the dynamics of spin transport and several experimental observables. To ease the following discussions, we first illustrate the density evolution in $\mathrm{Au}+\mathrm{Au}$ collisions at a beam energy of $100 \mathrm{MeV} /$ nucleon and an impact parameter of $b=8 \mathrm{fm}$ in Fig. 1 . In the present setup of projectile and target, a local polarization is observed and the participant (spectator) matter is slightly spin polarized in the $+y(-y)$ direction. However, one can estimate that this spin polarization leads to a negligible contribution to the time-even and time-odd spin-dependent mean-field potentials, compared to the contributions from the $\vec{h}_{2}$ and $\vec{h}_{3}$ expressed in Eqs. (8) and (9), with the latter displayed respectively in the third and fourth row in Fig. 1 . It is seen that the time-odd spin-dependent potential $\left(\vec{h}_{2}\right)$ is much stronger than the time-even one $\left(\vec{h}_{3}\right)$, giving spin-up (spin-down) nucleons a net attractive (repulsive) potential [33]. On the other hand, the $y$ component of the time-odd spin-dependent potential $\vec{h}_{2}$, i.e., $\nabla \times \vec{j}$, is two orders of magnitude larger than its $x$ and $z$ components. This justifies why we choose $y$ direction as the spin projection direction. 


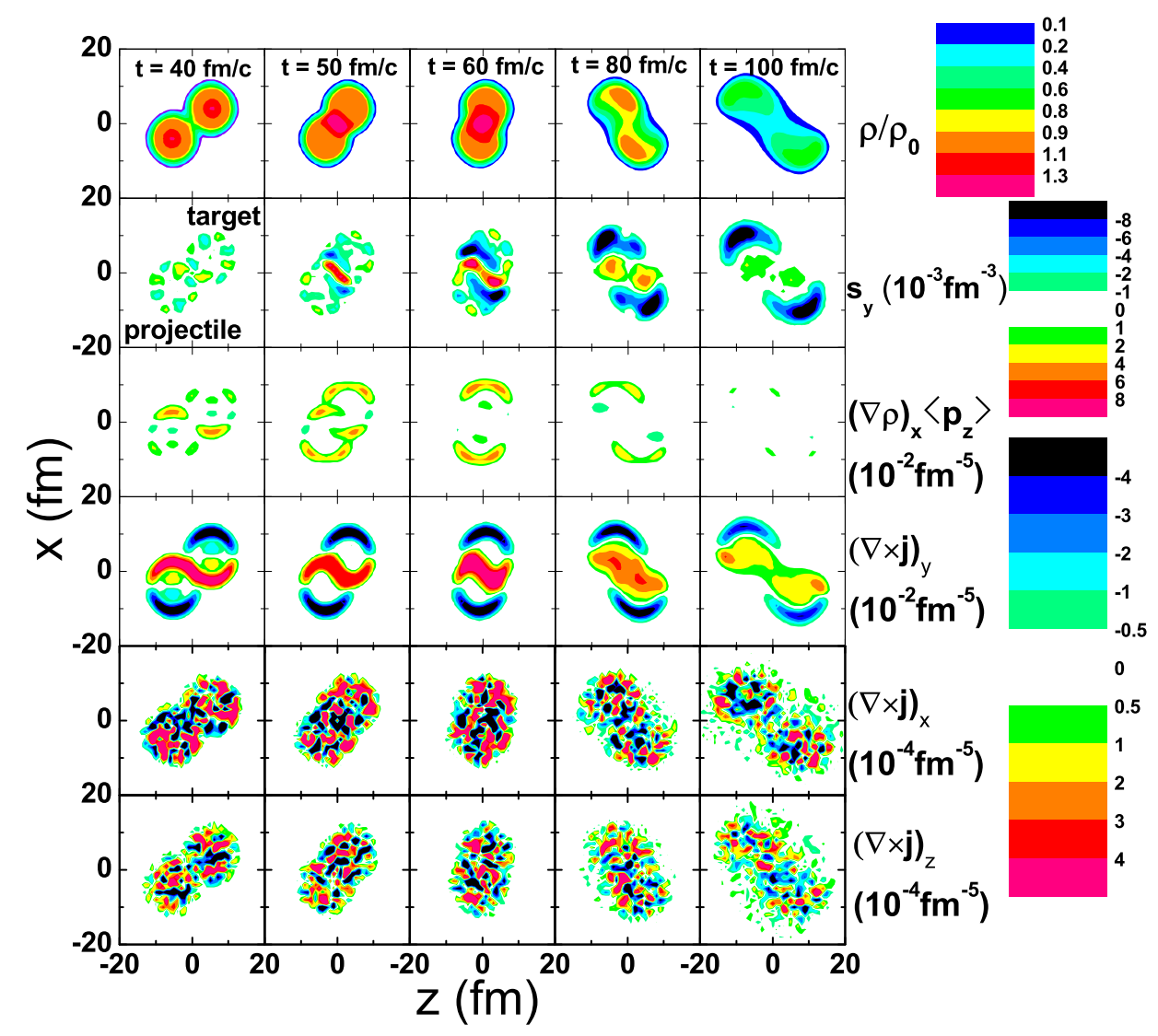

FIG. 1: (Color online) Contours of the nucleon reduced density $\rho / \rho_{0}$ (first row) with $\rho_{0}$ the saturation density, $y$ component of the spin density $s_{y}$ (second row), product of the $x$ component of the density gradient $(\nabla \rho)_{x}$ and the averaged $z$ component of nucleon momentum (third row), and the $y, x$, and $z$ component of the curl of the momentum density $(\nabla \times \vec{j})_{y}$ (fourth, fifth, and sixth row) in the reaction plane at different stages in $\mathrm{Au}+\mathrm{Au}$ collisions at a beam energy of 100 $\mathrm{MeV} /$ nucleon and an impact parameter of $\mathrm{b}=8 \mathrm{fm}$.

\section{A. Spin splitting of nucleon directed flow and elliptic flow}

We now investigate the spin-dependent nucleon collective flows. The distribution of nucleons with respect to the rapidity $y_{r}$ and transverse momentum $p_{T}$ in heavy-ion collisions can be expressed as $[34,35]$

$$
\frac{d^{3} N}{p_{T} d p_{T} d y_{r} d \phi}=\frac{d^{2} N}{2 \pi p_{T} d p_{T} d y_{r}}\left[1+2 v_{1}\left(y_{r}, p_{T}\right) \cos (\phi)+2 v_{2}\left(y_{r}, p_{T}\right) \cos (2 \phi)+\ldots\right],
$$

where $\phi=\tan ^{-1}\left(p_{y} / p_{x}\right)$ is the azimuthal angle,

$$
v_{1}=\langle\cos (\phi)\rangle=\left\langle\frac{p_{x}}{p_{T}}\right\rangle
$$


is the directed flow, and

$$
v_{2}=\langle\cos (2 \phi)\rangle=\left\langle\frac{p_{x}^{2}-p_{y}^{2}}{p_{x}^{2}+p_{y}^{2}}\right\rangle
$$

is the elliptic flow. Both the directed flow and the elliptic flow are important observables sensitive to the reaction dynamics and the equation of state of produced matter in heavy-ion collisions. In this study we investigate the collective flows of single nucleons that freeze out from the rest of the system at a local density less than $\rho_{0} / 8$.

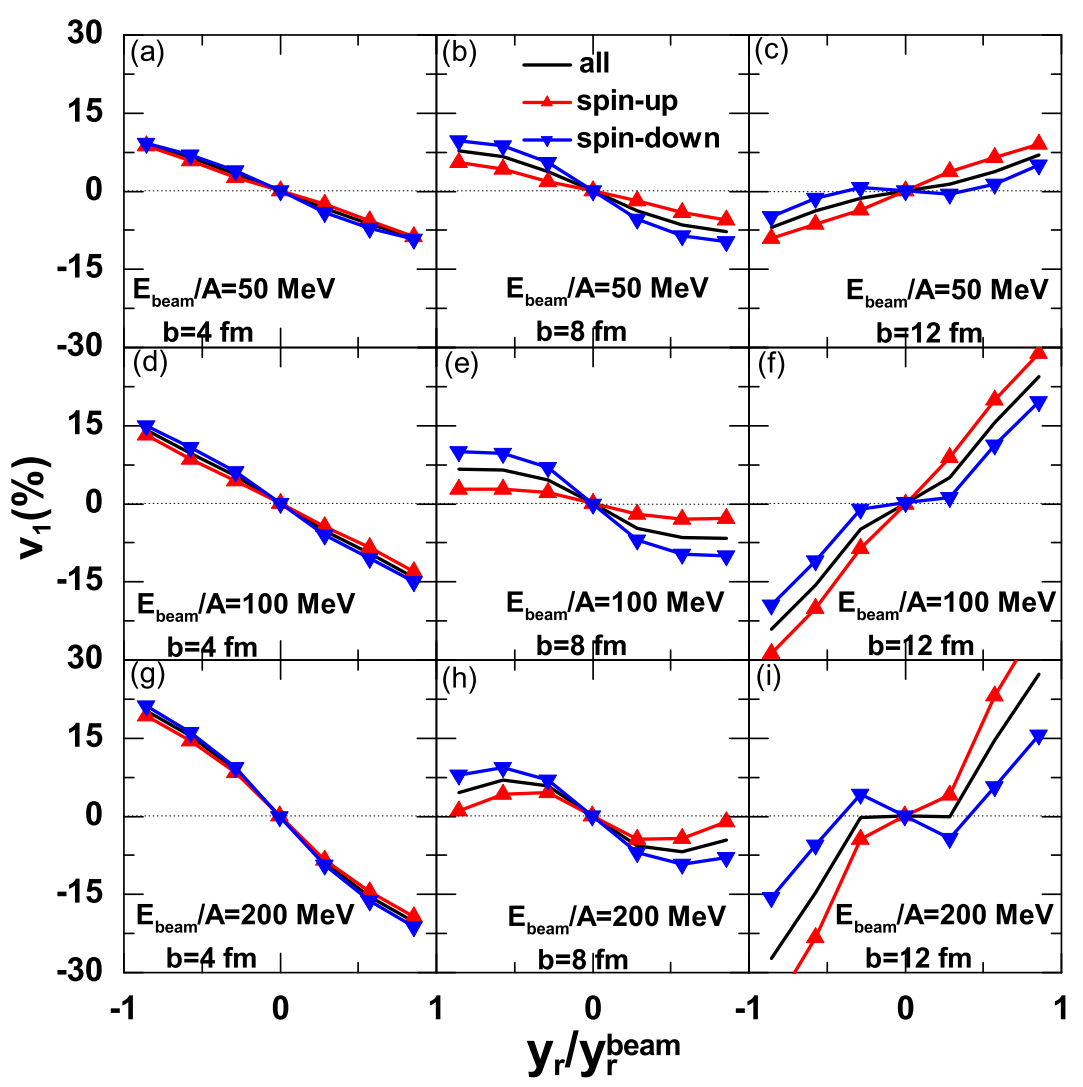

FIG. 2: (Color online) Directed flows for all free nucleons, spin-up nucleons, and spin-down nucleons as a function of reduced rapidity in $\mathrm{Au}+\mathrm{Au}$ collisions at different beam energies and impact parameters.

The directed flow characterizes the collective motion in the reaction plane. Figure 2 shows the directed flows of all nucleons as well as those for spin-up and spin-down nucleons in $\mathrm{Au}+\mathrm{Au}$ collisions at different beam energies and impact parameters, and the statistical errors are negligibly small if not shown. Because of different initializations of the projectile and the target in the momentum-coordinate space in simulations, the slope of the directed 
flow in our simulations might be opposite in sign compared to results obtained using other conventions of initializing nuclei. Due to the competition between the attractive mean-field potential in the energy range considered and the repulsive nucleon-nucleon scattering, the directed flow increases with increasing beam energy, and the slope is largest at $b=4 \mathrm{fm}$ and changes sign at $b=12 \mathrm{fm}$. Interestingly, one can observe clearly the flow splitting of spin-up and spin-down nucleons. The flow difference is mainly due to the residue potential from the cancellation of the time-even and the time-odd potential as discussed above, with spin-up (spin-down) nucleons affected by a more attractive (repulsive) potential and thus leading to a smaller (larger) or more (less) negative directed flow.

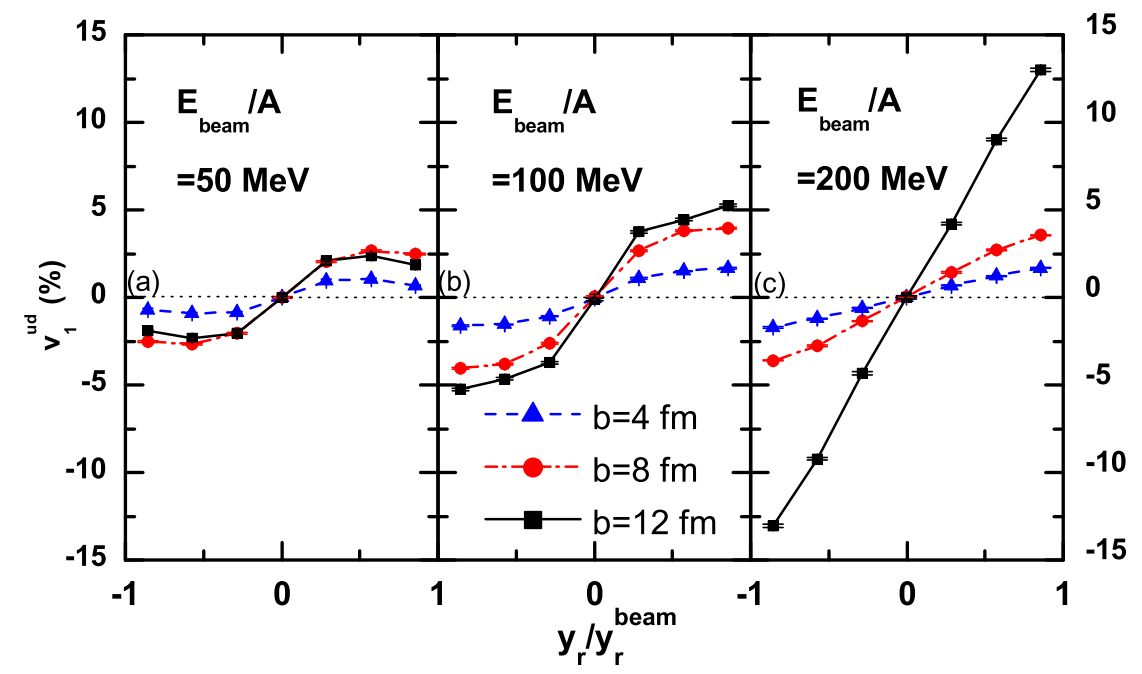

FIG. 3: (Color online) Spin up-down differential directed flows as a function of reduced rapidity in $\mathrm{Au}+\mathrm{Au}$ collisions at different beam energies and impact parameters.

In order to quantify the spin splitting of the directed flow, we defined the spin up-down differential directed flow as

$$
v_{1}^{u d}\left(y_{r}\right)=\frac{1}{N\left(y_{r}\right)} \sum_{i=1}^{N\left(y_{r}\right)} n_{i}\left(\frac{p_{x}}{p_{T}}\right)_{i},
$$

where $N\left(y_{r}\right)$ is the number of nucleons with rapidity $y_{r}$, and $n_{i}$ is $1(-1)$ for spin-up (spindown) nucleons. The above differential directed flow largely cancels the effect from spinindependent potentials while highlights the spin splitting of the flow as a result of spindependent potentials. Results for the spin up-down differential directed flow in $\mathrm{Au}+\mathrm{Au}$ collisions at different beam energies and different impact parameters are shown in Fig. 3. 
One sees from Figs. 2 and 3 that the magnitude of the differential directed flow can be as large as $50 \%$ of the total directed flow in some cases. On the other hand, the differential directed flow is generally stronger with increasing impact parameter, different from the total directed flow as show in Fig. 2. This is because the spin-dependent potentials are mostly related to the gradient of density or current, and it is thus a surface effect which is more important at larger impact parameters due to the diffusive density distribution of finite nuclei. In addition, as the angular momentum of heavy-ion collisions increases with increasing beam energy, the differential directed flow as a result of the nucleon spin-orbit coupling increases with increasing beam energy. On the other hand, the violent nucleon-nucleon scatterings, which play a more important role at higher collision energies, randomize the nucleon spin and thus wash out some of the effects from the spin-related potential. Therefore, the spin up-down differential directed flow becomes weaker at higher energies (not shown here). The competition between the above two effects leads to a maximum of the differential directed flow at beam energies around 100-200 MeV/nucleon in mid-central and peripheral $\mathrm{Au}+\mathrm{Au}$ collisions simulated using a spin-orbit coupling quadratic in momentum from the SHF approach. The energy range for the maximum spin up-down differential flow can be different if a different momentum dependence of the spin-orbit coupling is used.

We have also studied the system-size dependence of both the total directed flow and the spin up-down differential directed flow at different beam energies, and the results are shown in Fig. 4. Here we choose an impact parameter of $\mathrm{b}=8 \mathrm{fm}$ for ${ }^{197} \mathrm{Au}+{ }^{197} \mathrm{Au}$ collisions and $\mathrm{b}=6.9 \mathrm{fm}$ for ${ }^{124} \mathrm{Sn}+{ }^{124} \mathrm{Sn}$ collisions so that the two colliding systems have the same $\mathrm{b} / \mathrm{b}_{\max }$ and can be compared at the same centrality. It is seen that the directed flow is larger for ${ }^{197} \mathrm{Au}+{ }^{197} \mathrm{Au}$ collisions than for ${ }^{124} \mathrm{Sn}+{ }^{124} \mathrm{Sn}$ collisions at different beam energies due to the higher density reached in the heavier system and thus a higher pressure which leads to a larger directed flow. On the other hand, the spin up-down differential directed flow for ${ }^{197} \mathrm{Au}+{ }^{197} \mathrm{Au}$ collisions is slightly larger at the beam energies of 50 and $100 \mathrm{MeV} /$ nucleon and slightly smaller at the beam energy of $200 \mathrm{MeV} /$ nucleon than that for ${ }^{124} \mathrm{Sn}+{ }^{124} \mathrm{Sn}$ collisions. The similar spin up-down differential directed flow is due to the similar density gradient near the nucleus surface for ${ }^{197} \mathrm{Au}$ and ${ }^{124} \mathrm{Sn}$ as shown in the inset of Fig. 4, which leads to a similar strength of the spin-dependent potentials. It is thus seen that the spin splitting of the directed flow is a robust phenomena even in smaller systems.

The elliptic flow is a measure of the expansion of the almond-shaped medium formed in 


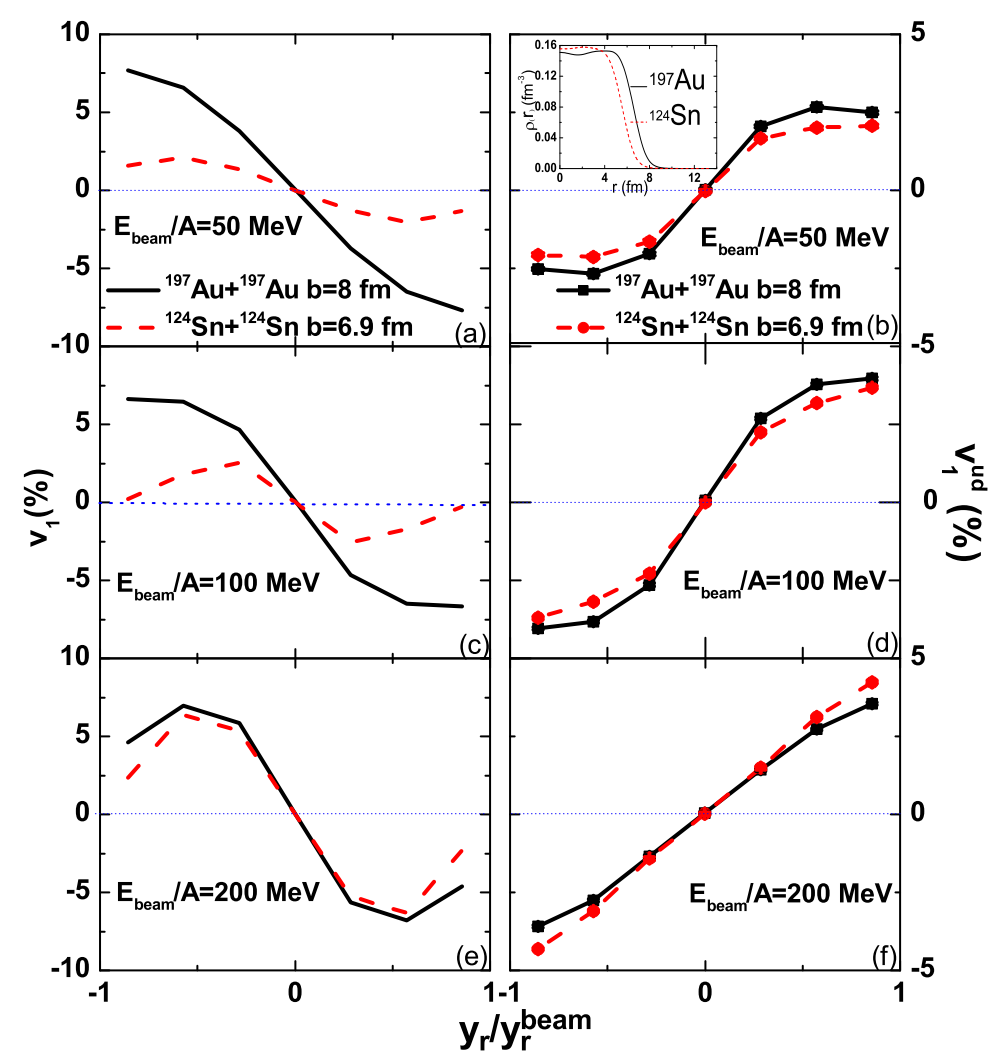

FIG. 4: (Color online) Directed flows (left column) and spin up-down differential directed flows (right column) as a function of reduced rapidity in mid-central ${ }^{197} \mathrm{Au}+{ }^{197} \mathrm{Au}$ and ${ }^{124} \mathrm{Sn}+{ }^{124} \mathrm{Sn}$ collisions at beam energies of 50,100, and $200 \mathrm{MeV} /$ nucleon at the same centrality. The density profiles for ${ }^{197} \mathrm{Au}$ and ${ }^{124} \mathrm{Sn}$ are shown in the inset.

heavy-ion collisions. It is a result of the competition between the squeeze-out flow perpendicular to the reaction plane and the in-plane flow. Its dynamics is thus more complicated than the directed flow itself. More quantitatively, the in-plane hydrodynamical flow and the out-of-plane squeeze-out flow contributes positively and negatively to the elliptic flow, respectively. Figure 5 displays respectively the elliptic flows of all free nucleons, spin-up nucleons, and spin-down nucleons in $\mathrm{Au}+\mathrm{Au}$ collisions at different beam energies and impact parameters. One sees that the sign of the elliptic flow changes from positive at lower energies to negative at higher energies. This is a result of blocking effects from the spectator on the expansion of the participant [36]. Moreover, it is seen that the energy at which the elliptic flow changes sign depends on the impact parameter. Similar to the case for the directed flow, the spin splitting of the elliptic flow is observed and the effect is more appreciable in 


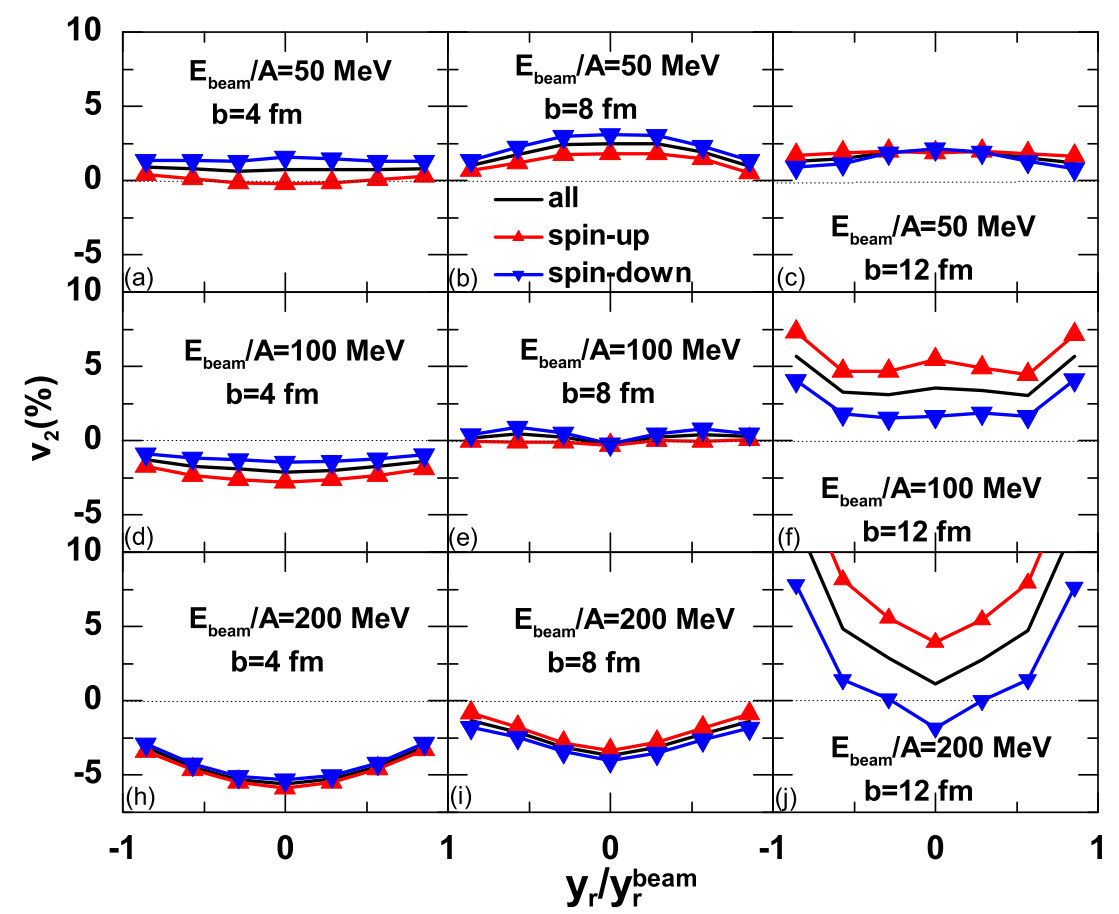

FIG. 5: (Color online) Elliptic flows of all free nucleons, spin-up nucleons, and spin-down nucleons in $\mathrm{Au}+\mathrm{Au}$ collisions at different beam energies and impact parameters.

peripheral collisions at beam energies of about 100-200 MeV/nucleon, from the SHF spinorbit coupling. However, the dynamics is more complicated as the magnitude of the elliptic flow depends on the nucleon potential as well as the shadowing from the spectator. In this case, a more attractive (repulsive) potential for spin-up (spin-down) nucleons somehow leads to a larger $v_{2}$. We have also observed that in this collision situation neutrons with more repulsive potential than protons have a smaller $v_{2}$ when the Coulomb potential is turned off, confirming the validity of our argument on the relative $v_{2}$ splitting and the potential difference.

To quantify the spin splitting of the elliptic flow, we can similarly define the spin up-down differential elliptic flow

$$
v_{2}^{u d}\left(y_{r}\right)=\frac{1}{N\left(y_{r}\right)} \sum_{i=1}^{N\left(y_{r}\right)} n_{i}\left(\frac{p_{x}^{2}-p_{y}^{2}}{p_{T}^{2}}\right)_{i},
$$

and the rapidity dependence of $v_{2}^{u d}$ is shown in Fig. 6. Consistent with Fig. 5, it is seen that the differential elliptic flow increases with increasing impact parameter and collision energy, and its magnitude can be as large as $50 \%$ of the total elliptic flow in peripheral collisions at 


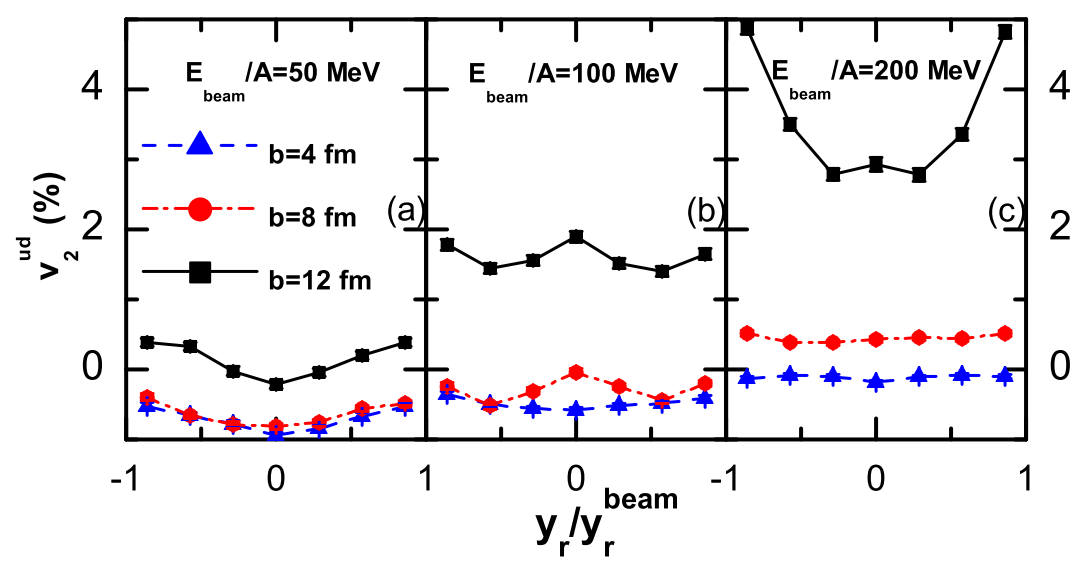

FIG. 6: (Color online) Spin up-down differential elliptic flow as a function of reduced rapidity in $\mathrm{Au}+\mathrm{Au}$ collisions at different beam energies and impact parameters.

beam energies of 100-200 MeV/nucleon.

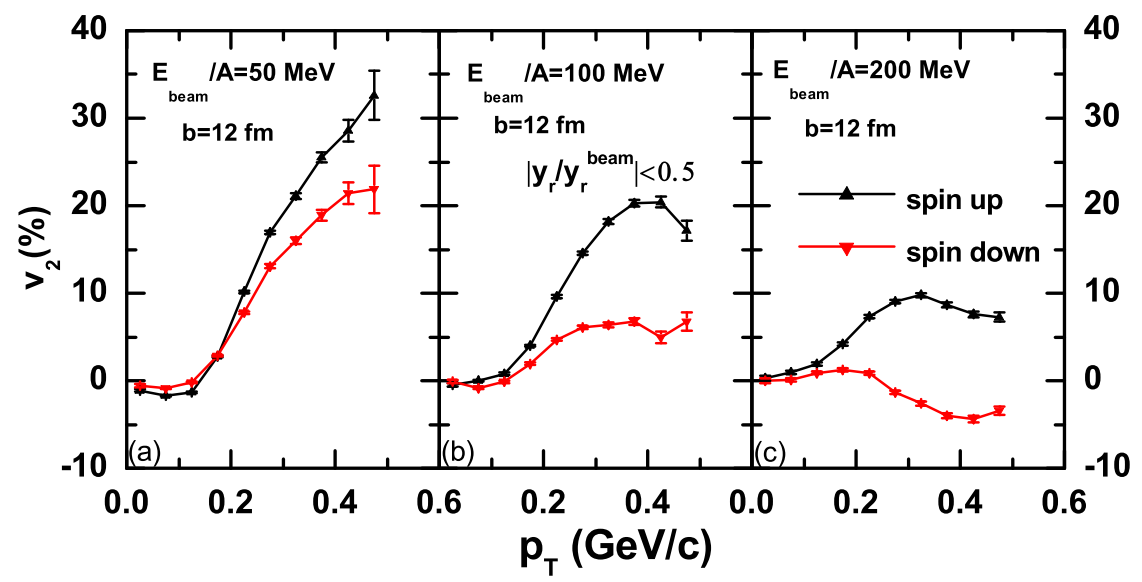

FIG. 7: (Color online) Transverse momentum dependence of the elliptic flow for mid-rapidity spin-up and spin-down nucleons in peripheral $\mathrm{Au}+\mathrm{Au}$ collisions at different beam energies.

We have also studied the transverse momentum dependence of the elliptic flow for midrapidity spin-up and spin-down nucleons, and the results in peripheral $\mathrm{Au}+\mathrm{Au}$ collisions at beam energies of 50, 100, and $200 \mathrm{MeV} /$ nucleon are shown in Fig. 7. The elliptic flow for spin-up nucleons are larger than that for spin-down nucleons, and the difference is larger at higher nucleon transverse momenta. This is understandable since the strength of the spin- 
related potentials increases with increasing nucleon momentum as one can see from Eqs. (8) and (9).

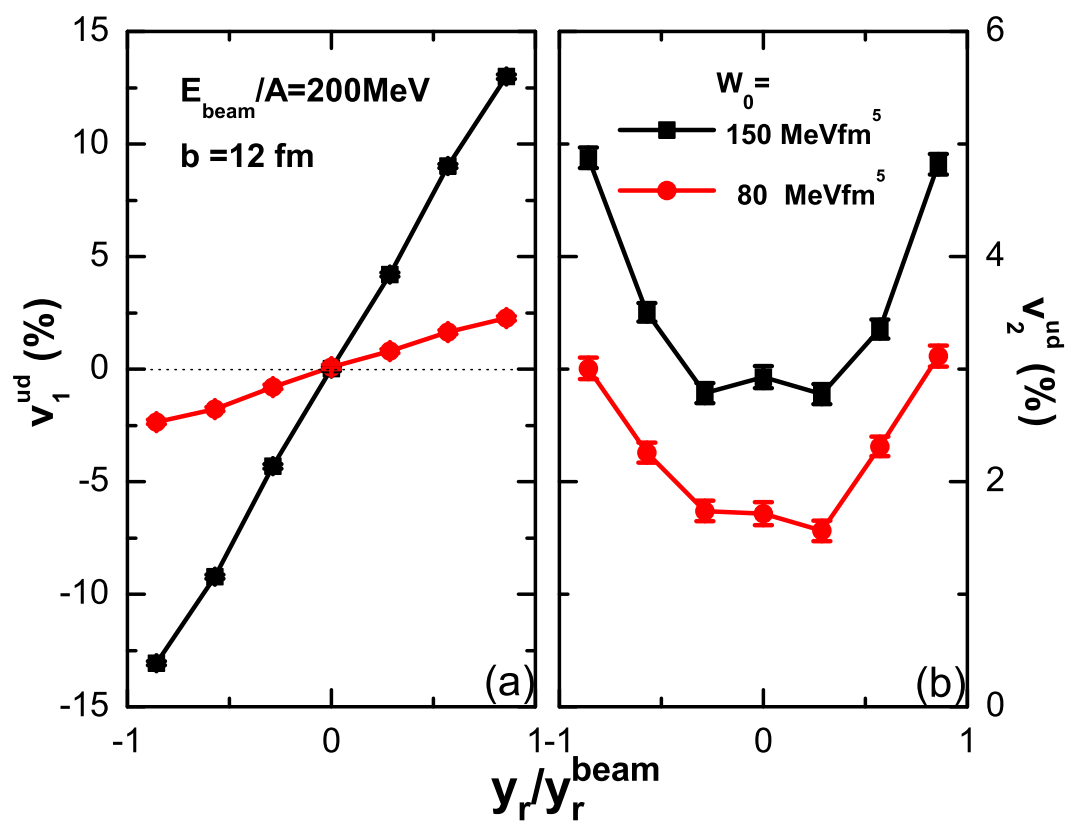

FIG. 8: (Color online) Spin up-down differential directed flow (a) and elliptic flow (b) with different spin-orbit coupling strength in peripheral $\mathrm{Au}+\mathrm{Au}$ collisions at a beam energy of $200 \mathrm{MeV} / \mathrm{nucleon}$.

The spin up-down differential flows defined above can be good probes of the nuclear spindependent interaction. As an illustration, the differential directed flows and elliptic flows from using different values of the nuclear spin-orbit coupling constant $W_{0}$ are compared in Fig. 8. It is seen that when the value of $W_{0}$ decreases from $150 \mathrm{MeVfm}^{5}$ to $80 \mathrm{MeVfm}^{5}$, the slope of the differential directed flow and the magnitude of the differential elliptic flow are also reduced by approximately a factor of five and two, respectively. It thus confirms that the spin-related mean-field potentials induced by the nuclear spin-dependent interaction are responsible for the spin dynamics of heavy-ion collisions. Both the spin up-down differential directed flow and elliptic flow are sensitive probes of the in-medium nuclear spin-orbit interaction and the dynamics of spin transport in intermediate-energy heavy-ion collisions. 


\section{B. Spin splitting of light cluster collective flows}

The present model can also be used to study spin-relevant observables of light clusters in intermediate-energy heavy-ion collisions. How to treat consistently the formation and dissociation of clusters especially the heavy ones dynamically in transport model simulations is still a challenging question. The phase-space coalescence model [37] is widely used as an afterburner of transport simulations to model cluster formation at the freeze-out stage of the reaction. The deexcitations of large and hot clusters is generally described by various statistical sequential decays or simultaneous multifragmentation models, such as the Gemini model [38] or the statistical multifragmentation model [39] (see, e.g., Ref. [40] and references therein). These hybrid models have been shown to be effective and reasonable in describing observables of light clusters, such as their multiplicities [41-45] and collective flows [4650]. In the present study, we adopt a dynamical coalescence model which evaluates the probability of producing a cluster by the overlap of its Wigner phase-space density with daughter nucleons $[45,47]$. This model is specially useful for studying light clusters which are loosely bound.

In previous studies, only a spin-independent Wigner function is used to calculate the Wigner phase-space density [45, 47] by using a statistical factor $G$ to take into account the spin-isospin degeneracy of light clusters. For example, the spin degeneracy factor for a proton and a neutron to form a deuteron is $3 / 8$. In our current model we can improve the dynamical coalescence with the explicit knowledge of the nucleon spin and isospin. The total wave function of light clusters is the direct product of spin, isospin, and coordinate space wave functions, and it must satisfy the antisymmetrization condition as a fermion system. If we assume that light clusters are in their ground states $(s$-wave with zero total angular momentum ${ }^{1}$, the product of spin and isospin wave function should be asymmetric.

Taking deuteron as an example, the various spin-isospin states for the combination of two nucleons are shown in Table I. From the direct product of spin and isospin wave function,

\footnotetext{
${ }^{1}$ We note that the angular momentum is not strictly conserved in most transport models so far but is violated within a few percentage.
} 
TABLE I: The spin wave function $\mid S>$ and isospin wave function $\mid I>$ for the combination of two nucleons with various spin-isospin states, with $S_{z}$ being the $z$ component of spin and $I_{3}$ being the third component of isospin.

\begin{tabular}{llll}
\hline $\mid S, S_{z}>$ Spin & Isospin & $\mid I, I_{3}>$ \\
\hline $\mid 1,1>$ & $\uparrow \uparrow$ & $p p$ & $\mid 1,1>$ \\
$\mid 1,0>$ & $\frac{1}{\sqrt{2}}(\uparrow \downarrow+\downarrow \uparrow)$ & $\frac{1}{\sqrt{2}}(p n+n p)$ & $\mid 1,0>$ \\
$\mid 1,-1>\downarrow \downarrow$ & $n n$ & $\mid 1,-1>$ \\
\hline $\mid 0,0>$ & $\frac{1}{\sqrt{2}}(\uparrow \downarrow-\downarrow \uparrow)$ & $\frac{1}{\sqrt{2}}(p n-n p)$ & $\mid 0,0>$ \\
\hline
\end{tabular}

we can get the eight wave functions for the combination of a proton and a neutron

$$
\begin{aligned}
\Psi_{1} & \sim \frac{1}{\sqrt{2}}\left(p^{\uparrow} n^{\uparrow}-n^{\uparrow} p^{\uparrow}\right), \\
\Psi_{2} & \sim \frac{1}{2}\left(p^{\uparrow} n^{\downarrow}+p^{\downarrow} n^{\uparrow}-n^{\uparrow} p^{\downarrow}-n^{\downarrow} p^{\uparrow}\right), \\
\Psi_{3} & \sim \frac{1}{\sqrt{2}}\left(p^{\downarrow} n^{\downarrow}-n^{\downarrow} p^{\downarrow}\right), \\
\Psi_{4} & \sim \frac{1}{2}\left(p^{\uparrow} n^{\downarrow}-p^{\downarrow} n^{\uparrow}-n^{\uparrow} p^{\downarrow}+n^{\downarrow} p^{\uparrow}\right), \\
\Psi_{5} & \sim \frac{1}{\sqrt{2}}\left(p^{\uparrow} n^{\uparrow}+n^{\uparrow} p^{\uparrow}\right), \\
\Psi_{6} & \sim \frac{1}{2}\left(p^{\uparrow} n^{\downarrow}+p^{\downarrow} n^{\uparrow}+n^{\uparrow} p^{\downarrow}+n^{\downarrow} p^{\uparrow}\right), \\
\Psi_{7} & \sim \frac{1}{\sqrt{2}}\left(p^{\downarrow} n^{\downarrow}+n^{\downarrow} p^{\downarrow}\right), \\
\Psi_{8} & \sim \frac{1}{2}\left(p^{\uparrow} n^{\downarrow}-p^{\downarrow} n^{\uparrow}+n^{\uparrow} p^{\downarrow}-n^{\downarrow} p^{\uparrow}\right) .
\end{aligned}
$$

As is known, the spin and isospin states are $S=1$ and $I=0$ for deuterons neglecting the approximately $4 \% d$-wave mixing. The wave function $\Psi_{1}, \Psi_{2}$, and $\Psi_{3}$ are thus feasible, and they happen to be the three possible spin states for deuterons, i.e., $S_{z}=-1,0$, and 1 . This is the reason why the statistical factor is $3 / 8$ for a proton and a neutron to form a deuteron. With the explicit knowledge of nucleon spin and isospin from the spin dynamics described in the present work, comparing the wave function $\Psi_{1}$ with $\Psi_{3}$ and $\Psi_{5}$ with $\Psi_{7}$, one can easily find that the statistical factor for a spin-up (spin-down) proton and a spin-up (spin-down) neutron to form a spin-up (spin-down) deuteron with $S_{z}=1\left(S_{z}=-1\right)$ is $1 / 2$. Similarly, the statistical factor is $1 / 4$ for a spin-up (spin-down) proton and a spin-down (spin-up) neutron to form a spin-aligned deuteron with $S_{z}=0$. 
TABLE II: Similar to Table I but for the combination of three nucleons.

\begin{tabular}{llll}
\hline $\mid S, S_{z}>$ & Spin & Isospin & $\mid I, I_{3}>$ \\
\hline $\mid 3 / 2,3 / 2>$ & $\uparrow \uparrow \uparrow$ & ppp & $\mid 3 / 2,3 / 2>$ \\
$\mid 3 / 2,1 / 2>$ & $\frac{1}{\sqrt{3}}(\uparrow \uparrow \downarrow+\downarrow \uparrow \uparrow+\uparrow \downarrow \uparrow)$ & $\frac{1}{\sqrt{3}}($ ppn + npp + pnp $)$ & $\mid 3 / 2,1 / 2>$ \\
$\mid 3 / 2,-1 / 2>$ & $\frac{1}{\sqrt{3}}(\downarrow \downarrow \uparrow+\uparrow \downarrow+\downarrow \uparrow \downarrow)$ & $\frac{1}{\sqrt{3}}($ nnp + pnn $+n p n)$ & $\mid 3 / 2,-1 / 2>$ \\
$\mid 3 / 2,-3 / 2>$ & $\downarrow \downarrow \downarrow$ & $n n n$ & $\mid 3 / 2,-3 / 2>$ \\
\hline $\mid 1 / 2,1 / 2>$ & $\frac{1}{\sqrt{6}}(2 \uparrow \uparrow \downarrow-\uparrow \downarrow \uparrow-\downarrow \uparrow \uparrow)$ & $\frac{1}{\sqrt{6}}(2 p p n-p n p-n p p)$ & $\mid 1 / 2,1 / 2>$ \\
$\mid 1 / 2,-1 / 2>$ & $\frac{1}{\sqrt{6}}(\uparrow \downarrow \downarrow+\uparrow \downarrow \uparrow-2 \downarrow \downarrow \uparrow)$ & $\frac{1}{\sqrt{6}}(p n n+n p n-2 n n p)$ & $\mid 1 / 2,-1 / 2>$ \\
\hline $\mid 1 / 2,1 / 2>$ & $\frac{1}{\sqrt{2}}(\uparrow \downarrow \uparrow-\downarrow \uparrow \uparrow)$ & $\frac{1}{\sqrt{2}}($ pnp $-n p p)$ & $\mid 1 / 2,1 / 2>$ \\
$\mid 1 / 2,-1 / 2>$ & $\frac{1}{\sqrt{2}}(\uparrow \downarrow \uparrow-\downarrow \uparrow \downarrow)$ & $\frac{1}{\sqrt{2}}($ pnn $-n p n)$ & $\mid 1 / 2,-1 / 2>$ \\
\hline
\end{tabular}

For triton and ${ }_{2}^{3} \mathrm{He}$ nucleus, it is very similar but a little more complicated. The various spin-isospin states for the combination of three nucleons are listed in Table II. Taking ${ }_{2}^{3} \mathrm{He}$ nuclei as an example, there are totally 24 combinations for two protons and one neutron. Considering the antisymmetrization of the wave function and the values of its spin and isospin, we finally find two wave functions satisfying all the conditions, i.e.,

$$
\begin{aligned}
& \Psi_{1}\left({ }_{2}^{3} \mathrm{He}\right) \sim \frac{1}{\sqrt{6}}\left(p^{\uparrow} n^{\uparrow} p^{\downarrow}-p^{\downarrow} n^{\uparrow} p^{\uparrow}-n^{\uparrow} p^{\uparrow} p^{\downarrow}+n^{\uparrow} p^{\uparrow} p^{\downarrow}-p^{\uparrow} p^{\downarrow} n^{\uparrow}+p^{\downarrow} p^{\uparrow} n^{\uparrow}\right), \\
& \Psi_{2}\left({ }_{2}^{3} \mathrm{He}\right) \sim \frac{1}{\sqrt{6}}\left(p^{\uparrow} n^{\downarrow} p^{\downarrow}-p^{\downarrow} n^{\downarrow} p^{\uparrow}-n^{\downarrow} p^{\uparrow} p^{\downarrow}+n^{\downarrow} p^{\uparrow} p^{\downarrow}-p^{\uparrow} p^{\downarrow} n^{\downarrow}+p^{\downarrow} p^{\uparrow} n^{\downarrow}\right) .
\end{aligned}
$$

Thus, the statistical factor is $1 / 12$ without knowing the information of nucleon spin. The statistical factor is $1 / 6^{2}$ for a spin-up (spin-down) neutron, a spin-up proton, and a spindown proton to form a spin-up (spin-down) ${ }_{2}^{3} \mathrm{He}$ nucleus with $S_{z}=1 / 2\left(S_{z}=-1 / 2\right)$, comparing $\Psi_{1}\left({ }_{2}^{3} \mathrm{He}\right)$ or $\Psi_{2}\left({ }_{2}^{3} \mathrm{He}\right)$ with the other 5 spin-isospin states having the same spin and isospin values of nucleons but not satisfying the antisymmetrization condition. The procedure is similar for tritons, and it can be found that the statistical factor is also $1 / 6$ for a spin-up (spin-down) proton, a spin-up neutron, and a spin-down neutron to form a spin-up (spin-down) triton with $S_{z}=1 / 2\left(S_{z}=-1 / 2\right)$.

Using the improved dynamical coalescence model coupled to the SIBUU12 transport model, we can study various spin-relevant observables of light clusters. Here we focus on the spin-dependent collective flows of light clusters. Figure 9 displays directed flows for

\footnotetext{
${ }^{2}$ It changes to $1 / 3$ if the order of another two neutrons with spin-up and spin-down is not specified.
} 


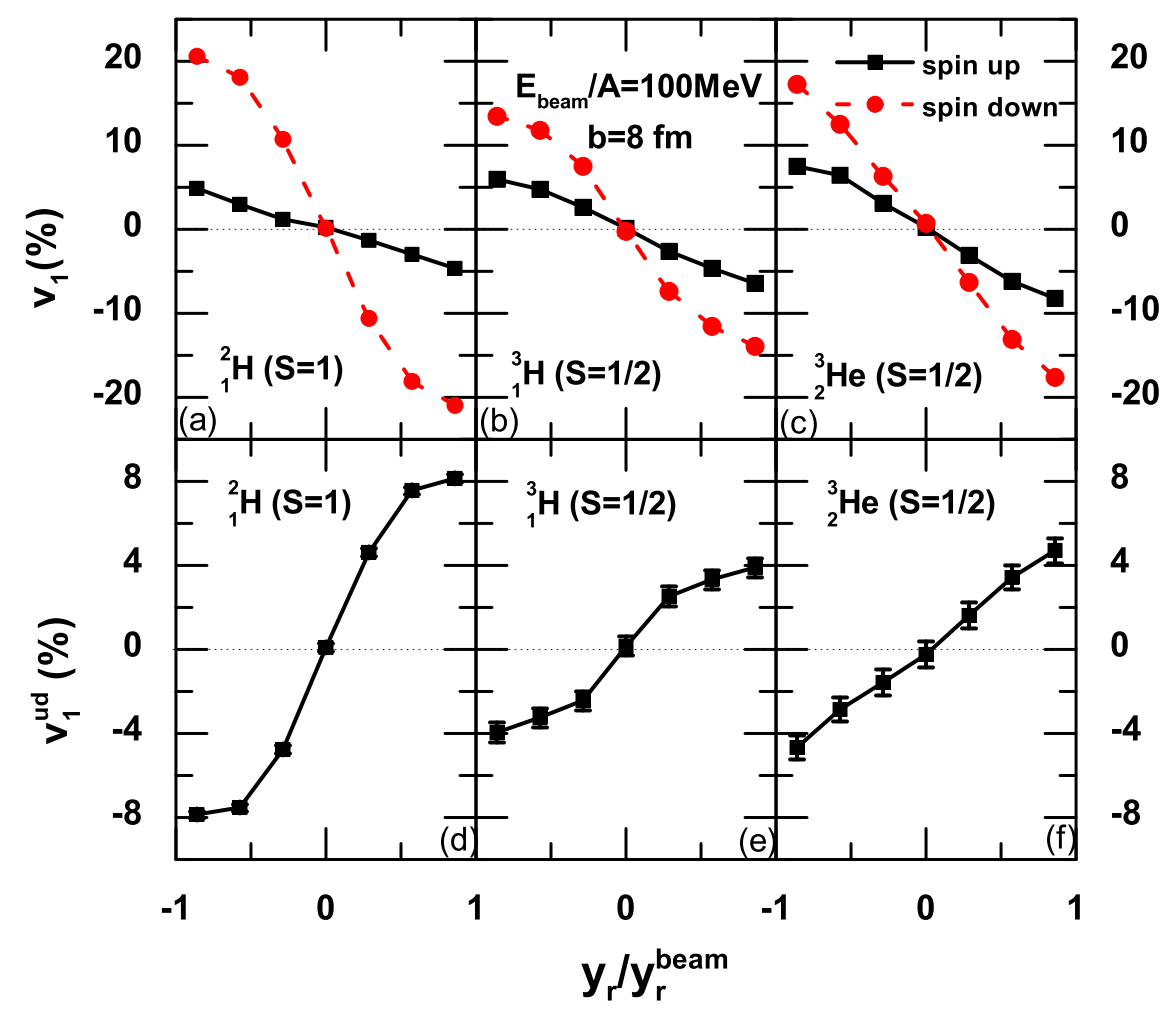

FIG. 9: (Color online) Directed flows (upper panels) and spin up-down differential directed flows (lower panels) of deuterons ((a) and (d)), tritons ((b) and (e)), and ${ }_{2}^{3} \mathrm{He}((\mathrm{c})$ and (f)) for their different spin states in mid-central $\mathrm{Au}+\mathrm{Au}$ collisions at a beam energy of $100 \mathrm{MeV} /$ nucleon.

different spin states of deuterons, tritons, and ${ }_{2}^{3} \mathrm{He}$ as well as the corresponding spin updown differential directed flows. The largest spin splitting of deuteron directed flow and the corresponding spin up-down differential directed flow is observed, compared with that for tritons and ${ }_{2}^{3} \mathrm{He}$. This is understandable since deuteron is a spin-1 particle and a deuteron with $S_{z}=1\left(S_{z}=-1\right)$ is formed by a neutron and a proton with both $S_{z}=1 / 2\left(S_{z}=-1 / 2\right)$, while a spin-up (spin-down) triton or ${ }_{2}^{3} \mathrm{He}$ nucleus is formed by two spin-up (spin-down) nucleons and one spin-down (spin-up) one. The spin splitting of elliptic flows for deuterons, tritons, and ${ }_{2}^{3} \mathrm{He}$ is further shown in Fig. 10. The difference of elliptic flows between different spin states is larger at large backward and forward rapidities. Again, the spin splitting of elliptic flows is more obvious for deuterons considering the statistical error bars, compared with that for tritons and ${ }_{2}^{3} \mathrm{He}$ nuclei. Thus, deuterons can be a good probe of the nuclear spin-orbit coupling in intermediate-energy heavy-ion collisions if the different spin states can 
be identified experimentally.

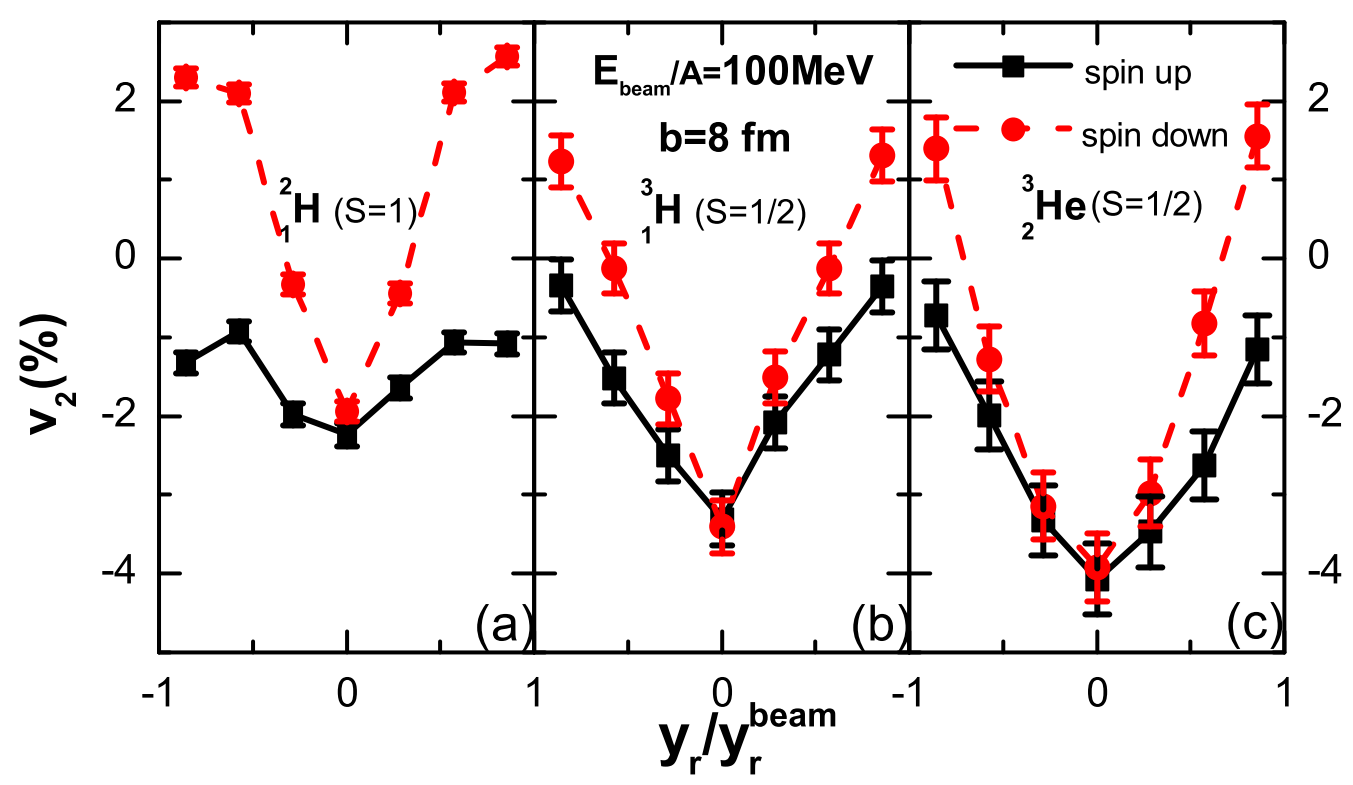

FIG. 10: (Color online) Elliptic flows of deuterons (a), tritons (b), and ${ }_{2}^{3} \mathrm{He}$ (c) for their different spin states as a function of reduced rapidity in mid-central $\mathrm{Au}+\mathrm{Au}$ collisions at a beam energy of $100 \mathrm{MeV} /$ nucleon.

\section{CONCLUSION AND OUTLOOK}

Within the spin- and isospin-dependent Boltzmann-Uehing-Uhlenbeck transport model (SIBUU12) which takes into account explicitly nucleon spin states and a Skyrme-type spinorbit interaction, we have investigated the dynamics of spin transport in intermediate-energy heavy-ion collisions. To study spin-related observables of light clusters, we extended the dynamical coalescence model by considering the nucleon spin explicitly. Several collective observables and their dependence on the strength of the spin-orbit coupling are examined. In particular, the spin splitting of both the directed flow and the elliptic flow are found to maximize in $\mathrm{Au}+\mathrm{Au}$ reactions at beam energies of about $100-200 \mathrm{MeV} /$ nucleon, and they are generally more appreciable in peripheral collisions. The effect is also considerable in smaller collision systems and becomes larger for energetic nucleons. The spin up-down differential directed flow and elliptic flow for both nucleons and light clusters are found to be sensitive 
to and robust probes of the in-medium nuclear spin-orbit interactions. Overall, our study sheds new light on the dynamics of spin transport in heavy-ion collisions at intermediate energies and possible observables to probe experimentally the in-medium spin-orbit coupling relevant for resolving some interesting problems in both nuclear structure and astrophysics.

The spin splitting from the present study is much larger than that from our previous studies [20-22]. The results presented in this manuscript represent the upper limit of the spin effect in intermediate-energy heavy-ion collisions. The nuclear spin-orbit coupling plays the similar role of an external magnetic field as that in the Stern-Gerlach experiments. In this sense, the present method describes properly the correlation between the spin and trajectory, while the previous method takes the nucleon spin evolution in the mean field into consideration. It is still a challenge to treat consistently both the nucleon spin evolution in the mean field as well as the spin-trajectory correlation in the spin dynamics of intermediateenergy heavy-ion collisions.

Further studies and improvements can be carried out based on the present framework in order to explore interesting topics of spin dynamics in heavy-ion collisions. For example, the effects of shell structure and the total spin of a nucleus can be further incorporated in the initialization. The former is related to the correlation between the nucleon phase-space distribution and its spin, and the latter, which is the sum of orbital and spin angular momenta of all the nucleons, can be important if spin polarized nuclei can be used in terrestrial laboratories. The combined effects of the nuclear spin-orbit coupling and the magnetic field, with the latter also perpendicular to the reaction plane, can be further explored at different beam energies. Furthermore, the spin-dependent nucleon-nucleon scattering cross sections as well as the nuclear tensor force may be important ingredients of spin dynamics. Such studies are in progress.

\section{Acknowledgments}

We thank Chen Zhong for maintaining the high-quality performance of the computer clusters for the simulation of the present work, Wolfgang Trautmann for helpful discussions on the possible ways of measuring nucleon spin, and the anonymous referee for providing useful comments and suggestions with great patience. This work was supported by the Major State Basic Research Development Program (973 Program) of China under Con- 
tract Nos. 2015CB856904 and 2014CB845401, the National Natural Science Foundation of China under Grant Nos. 11320101004, 11475243, and 11421505, the "100-talent plan" of Shanghai Institute of Applied Physics under Grant Nos. Y290061011 and Y526011011 from the Chinese Academy of Sciences, the Shanghai Key Laboratory of Particle Physics and Cosmology under Grant No. 15DZ2272100, the "Shanghai Pujiang Program" under Grant No. 13PJ1410600, the US National Science Foundation under Grant No. PHY-1068022, the U.S. Department of Energy, Office of Science, under Award Number de-sc0013702, and the CUSTIPEN (China-U.S. Theory Institute for Physics with Exotic Nuclei) under the US Department of Energy Grant No. DEFG02-13ER42025.

[1] B. A. Li, C. M. Ko, and W. Bauer, Int. J. Mod. Phys. E 7, 147 (1998).

[2] Isospin Physics in Heavy-Ion Collisions at Intermediate Energies, edited by B. A. Li and W. Udo Schroeder (Nova Science, New York, 2001).

[3] V. Baran, M. Colonna, V. Greco, and M. Di Toro, Phys. Rep. 410, 335 (2005).

[4] A. W. Steiner, M. Prakash, J. M. Lattimer, and P.J. Ellis, Phys. Rep. 411, 325 (2005).

[5] J. M. Lattimer and M. Prakash, Phys. Rep. 442, 109 (2007).

[6] B. A. Li, L. W. Chen, and C. M. Ko, Phys. Rep. 464, 113 (2008).

[7] "Topical issue on nuclear symmetry energy", Eds., B. A. Li, A. Ramos, G. Verde, and I. Vidaña, Eur. Phys. J. A 50, No. 2 (2014).

[8] M. Goeppert-Mayer, Phys. Rev. 75, 1969 (1949).

[9] O. Haxel et al., Phys. Rev. 75, 1766 (1949).

[10] M. M. Sharma et al., Phys. Rev. Lett. 74, 3744 (1995).

[11] J. M. Pearson and M. Farine, Phys. Rev. C 50, 185 (1994).

[12] P. G. Reinhard and H. Flocard, Nucl. Phys. A 584, 467 (1995).

[13] R. J. Furnstahl, John J. Rusnak, and Brian D. Serot, Nucl. Phys. A 632, 607 (1998).

[14] M. Bender et al., Phys. Rev. C 60, 034304 (1999).

[15] J. P. Schiffer et al., Phys. Rev. Lett. 92, 162501 (2004) [Erratum-ibid 110, 169901 (2013)].

[16] L. Gaudefroy et al., Phys. Rev. Lett. 97, 092501 (2006).

[17] Y. Ichikawa et al., Nature Phys. 8, 918 (2012).

[18] https://wiki.bnl.gov/rhicspin/Presentations 
[19] A. Zelenski et al., J. Phys: Conf. Ser. 295, 012132 (2011).

[20] J. Xu and B. A. Li, Phys. Lett. B 724, 346 (2013).

[21] Y. Xia, J. Xu, B. A. Li, and W. Q. Shen, Phys. Rev. C 89, 064606 (2014).

[22] J. Xu, B. A. Li, W. Q. Shen, and Y. Xia, Front. Phys. 10, 102501 (2015).

[23] C. C. Guo, Y. J. Wang, Q. F. Li, and F. S. Zhang, Phys. Rev. C 90, 034606 (2014).

[24] Y. Xia, J. Xu, B. A. Li, and W. Q. Shen, arXiv: 1602:00404v2 [nucl-th].

[25] H. Smith and H. H. Jensen, Transport Phenomena (Oxford University press, Oxford, 1989).

[26] P. Ring and P. Schuck, The Nuclear Many-Body Problem (Springer, Berlin, 1980).

[27] E. B. Balbutsev, I. V. Molodtsova, and P. Schuck, Phys. Rev. C 88, 014306 (2013).

[28] D. Vautherin and D. M. Brink, Phys. Rev. C 5, 626 (1972).

[29] Y. M. Engle et al., Nucl. Phys. A 249, 215 (1975).

[30] P. G. Reihard, Rep. Prog. Phys. 52, 439 (1989).

[31] C. Y. Wong, Phys. Rev. C 25, 1460 (1982).

[32] L. W. Chen et al., Phys. Rev. C 82, 024321 (2010).

[33] According to the present configuration of projectile and target, the total angular momentum of the system is in the $+y$ direction. If initially the target (projectile) nucleus is put on the $-x(+x)$ side like the convensional configuration used by others in non-central collisions, the total angular momentum is in the $-y$ direction. In that case the spin-up (spin-down) nucleons with spin in the $+y(-y)$ direction have a net repulsive (attractive) potential as a result of the spin-orbit coupling.

[34] J. Y. Ollitrault, Phys. Rev. D 46, 229 (1992).

[35] A. M. Poskanzer and S. A. Voloshin, Phys. Rev. C 58, 1671 (1998).

[36] P. Danielewicz, R. Lacey, and W. G. Lynch, Science 298, 1592 (2002).

[37] P. E. Hodgson and E. Běták, Phys. Rep. 374, 1 (2003).

[38] R. J. Charity et al., Nucl. Phys. A 483, 371 (1988).

[39] J. P. Bondorf, A. S. Botvina, A. S. Iljinov, I. N. Mishustin, and K. Sneppen, Phys. Rep. 257, 133 (1995).

[40] M. B. Tsang et al., Eur. J. Phys. A 30, 129 (2006).

[41] H. Kruse, B. V. Jacak, J. J. Molitoris, G. D. Westfall, and H. Stöcker, Phys. Rev. C 37, 1770 (1985).

[42] B. A. Li, A. R. DeAngeli, and D. H. E. Gross, Phys. Lett. B 303, 225 (1993). 
[43] K. Hagel et al., Phys. Rev. C 62, 034607 (2000).

[44] W. P. Tan et al., Phys. Rev. C 64, 051901(R) (2001).

[45] L. W. Chen, C. M. Ko, and B. A. Li, Phys. Rev. C 68, 017601 (2003); Nucl, Phys. A 729, 809 (2003).

[46] V. Koch et al., Phys. Lett. B 241, 174 (1990).

[47] R. Mattiello et al., Phys. Rev. Lett. 74, 2180 (1995); Phys. Rev. C 55, 1443 (1997).

[48] L. W. Chen, F. S. Zhang, and G. M. Jin, Phys. Rev. C 58, 2283 (1998).

[49] F. S. Zhang, L. W. Chen, Z. Y. Ming, and Z. Y. Zhu, Phys. Rev. C 60, 064604 (1999).

[50] G. C. Yong, B. A. Li, L. W. Chen, and X. C. Zhang, Phys. Rev. C 80, 044608 (2009). 